Apidologie, 1973, 4 (1), 1-24.

\title{
RASSENSPEZIFISCHE UNTERSCHIEDE IM LERNVERHALTEN DER HONIGBIENE (APIS MELLIFICA L.)*
}

\section{Différences intraspécifiques dans le comportement d'apprentissage chez l'abeille (Apis mellifica L.)}

R. MENZEL, H. FREUDEL und U. RÜHL

Fachbereich Biologie - Zoologie - Technische Hochschule Darmstadt

\section{SUMMARY}

INTRASPECIFIC DIFFERENCES IN THE LEARNING BEHAVIOUR OF THE HONEY BEE (Apis mellifica L.)

Three subspecies of Apis mellifera (carnica, ligustica, lamarckii (earlier formerly fasciata)) and the species Apis cerana are compared concerning their learning behaviour in two experimental arrangements (horizontal and vertical color crosses, fig. 1). The learning process is represented by learning curves, which separately interpret the decisions made on approach (A), on touching the mark (T) and on alighting (S). The $T+S$ curves are steeper than the $A$ curves of all subspecies (compare fig. 2 and 6 with 3 and 4). A.m. lamarckii excepted, all A curves for the vertical yellow cross are steeper than for the horizontal yellow cross.

Apis cerana learns the quickest and reaches the highest level; A.m. lamarckii shows a similar steep learning curve (fig. 3,4). Both species accomodate their strategy of search in such a way that in the learning process more certain decisions ( $T$ and $S$ ) get more frequent (fig. 5). The flatter A curves of A.m. lamarckii with vertical crosses (fig. 4) can be interpreted as an alteration in the linkage of the percentage of correct $A$ choices with correct $T+S$ choices (fig. 6, 7), A.m. carnica and A.m. ligustica have flatter A curves (fig. 3, 4). They do not improve their strategy of search in the learning process (fig. 5). With both species markedly

* Mit Unterstützung durch Mittel der Deutschen Forschungsgemeinschaft, die Herrn Prof. Dr. F. Rutron zur. Verfügung standen. Wir danken Herrn Prof. Rutrser herzlich für seine Hilfe. Herrn MasuHR danken wir für die kritische Durchsicht des Manuskripts.

Staatsexamensarbeit für das höhere Lehramt im Fachbereich Biologie der TH Darmstadt. 
A.m. ligustica the linkage of the percentage of correct $\mathbf{A}$ choices with correct $\mathbf{T}+\mathbf{S}$ choices changes in such a way that the vertical yellow cross is learned better than the horizontal one (fig. 6).

The motivation of search, measured by approaches per minute, differs specifically in the 4 subspecies (fig. 9).

In another series of tests with A.m. carnica and A.m. ligustica, we can show that spatial parameters for both of them are of great importance in case of vertical marks than of horizontal ones. A.m. ligustica learns the spatial relationship of marks better than A.m. carnica.

In discussing the results, we can show that statements concerning the mechanisms of learning can be made only if the tendency of spontaneous choice is known before the learning process starts. Besides, we must take into account that the complex behaviour of choice changes during the learning process and differs in various test situations. of view.

The differences stated between the subspecies are shortly considered from ecological points

\section{ZUSAMMENFASSUNG}

Drei Rassen von $A$. mellifica (carnica, ligustica, lamarckii $=$ früher fasciata) und Apis cerana werden an 2 Versuchsanordnungen (horizontal und vertikal angeordnete Farbkreuze, Abb. 1) vergleichend auf ihr Lernverhalten untersucht. Der Lernfortschritt wird mit Lernkurven beschrieben. Das Wahlverhalten wird in An flugs- (A), Tipp- (T) und Sitz-(S) Entschejdungen unterschieden. Die T + S-Lernkurven sind bei allen Rassen steiler als die A-Lernkurven (vgl. Abb. 2 und 6 mit 3 und 4). Mit Ausnahme der A-Lernkurve von A. m. lamarckii sind die Lernkurven für das vertikale Gelbkreuz stets steiler als für das horizontale.

$A$. cerana lernt am schnellsten und erreicht das höchste Lernniveau; A.m. lamarckii zeigt einen ähnlich steilen Lernverlauf (Abb. 3,4). Beide Rassen verändern ihre Suchstrategie im Lernverlauf so, daß sicherere Entscheidungen ( $T$ und $S$ ) häufiger auftreten (Abb. 5). Der flachere Verlauf der A-Lernkurve von A.m. lamarckii bei vertikalen Marken (Abb. 4) läßt sich auf eine Veränderung der Kopplung zwischen $A-W a h l t e n d e n z$ und $T+S-W a h l t e n d e n z$ zurückführen (Abb. 6,7). A.m. carnica und ligustica weisen flachere A-Lernkurven auf (Abb. $3,4)$. Sie verbessern ihre Suchstrategie im Lernverlauf nicht (Abb. 5). Bei beiden Rassen besonders ausgeprägt aber bei A.m. ligustica - verändert sich die Kopplung zwischen AWahltendenz und $\mathrm{T}+\mathrm{S}$-Wahltendenz im Lernverlauf so, daß das vertikale Gelbkreuz besser gelernt wird als das horizontale (Abb. 6). Die Wahlmotivation (An flüge pro Testminute) ist bei $A$.m. carnica und ligustica anfänglich hoch und nimmt im Lernverlauf ab, bei $A$.m. lamarckii und $A$. cerana niedrig und ändert sich wenig. (Abb. 9).

In einer 2. Versuchsserie mit A.m. carnica und A.m. ligustica wird gezeigt, daß Raumparameter für beide Rassen auf der vertikalen Anordnung ein größeres Gewicht haben als auf der horizontalen. Von A.m. ligustica wird die Lage der Lernzeichen im Raum noch besser gelernt als von $A . m$. carnica.

In der Diskussion der Ergebnisse wird gezeigt, daß nur dann mit Lernkurven Aussagen über den Lernmechanismus gemacht werden können, wenn die Wahltendenz jedes Versuchstieres vor Lernbeginn bekannt ist und wenn berücksichtigt wird, daß sich das Wahlverhalten im Lernverlauf und in verschiedenen Versuchssituationen qualitativ verändern kann; diese Veränderung muß bei der Interpretation der Lernkurven berücksichtigt werden. Auf ökologische Gesichtspunkte wird kurz hingewiesen.

Um die mannigfaltigen Reize aus der Umwelt zu verarbeiten, stehen den Tieren 2 interne Informationsquellen zur Steuerung ihres Verhaltens zur Verfügung : das genetisch fixierte Verhaltensinventar, das sich im Verlauf phylogenetischer Anpassung der Art herausgebildet hat (niedergelegt im ( Artgedächtnis ») und die durch Erfahrung des einzelnen Tieres mit der wechselvollen Umwelt erfolgte Wertung möglicher Verhaltensweisen (gespeichert im ( Individualgedächtnis »). Das Artgedächtnis umfaßt das Spek- 
trum der möglichen Verhaltensweisen, das Individualgedächtnis die Bedeutung einzelner Aktionen und Reaktionen. Die Mechanismen zur Bildung dieser beiden Informationsquellen und ihr stoffliches Substrat sind wesentlich verschieden. Dennoch wirken beide auf noch unbekannte Weise auf die neuronale Musterbildung ein. Ein biologischer Zugang zu dem komplexen Problem des individuellen Lernens könnte daher die Analyse der gegenseitigen Abhängigkeit von Artgedächtnis und Individualgedächtnis sein, zumal die Molekulargenetik in den letzten Jahren tiefe Einblicke in die genetischen Steuerungsmechanismen gebracht hat. Eine solche Analyse muß mit kleinen Schritten beginnen, z.B. dem Abtasten des genetischen Rahmens, in dem individuelles Lernen möglich ist, also der Beschreibung der Reaktionsnorm von Lernmechanismen.

Da Insekten in ihrem Verhalten weitgehend instinktgebunden sind (Steuerung über das Artgedächtnis) und nur an einigen exponierten Stellen Lernen notwendig und möglich ist, eignen sie sich für eine solche Analyse besonders. Bienen bieten sich als Versuchstiere an, weil sie bei der Nahrungssuche Orientierungsmarken lernen müssen, solche Orientierungsmarken sich sauber beschreiben lassen, die Versuchstiere mit Suchmotivation freiwillig in den Lerntest kommen, beliebig viele Versuchstiere mit identischer genetischer Ausstattung untersucht werden können und einige verschiedene Rassen zur Verfügung stehen (RuTtNeR, 1967).

\section{METHODE}

Aufgrund der vielfältigen Parameter, die den Lernvorgang beein flussen und vom Experimentator nur zum Teil wirklich quantitativ erfaßt werden können, spielt die gewählte Methode und die Art der Versuchsauswertung eine große Rolle. Deshalb soll hier die Methode etwas ausführlicher beschrieben werden.

Die Experimente wurden im Sommer 1970 und 1971 mit den Bienenrassen A.m. carnica, A. m. ligustica, A. m. lamarckii (früher $A$. m. fasciata) und der Art $A$. cerana durchgeführt. Reinrassige Völker stellte uns Herr Prof. F. Rutrner zur Verfügung. Die indische Biene stammte vom Khaiber-Paß (1 $028 \mathrm{~m}$ ) und war im gleichen Jahr von dort geholt worden.

Die Flugstrecke zwischen Stock und Futterquelle hat für die verschiedenen Rassen subjektiv unterschiedliche Bedeutung, wie man aus dem Übergang zu gerichteten Schwänzeltänzen entnehmen kann (v. FRISCH, 1965). Wir haben versucht, dies auszugleichen, indem wir die Entfernung zur Versuchsanordnung so wählten, daß Neulinge stets über Schwänzeltänze alarmiert wurden, und haben daher für die verschiedenen Rassen unterschiedliche Entfernungen zur Dressuranordnung gewählt : A.m. carnica und ligustica $: 180 \mathrm{~m}$ (in NW-Richtung), A.m. lamarckii und $A$. cerana $: 30 \mathrm{~m}$ (in O-Richtung).

Die beiden Versuchsanordnungen, an denen gleichzeitig gearbeitet wurde, standen jeweils auf einer Wiese; die Entfernung zu den nächsten Landmarken (niedrige Büsche) betrug mind. $15 \mathrm{~m}$. Die horizontale Anordnung bestand aus einem runden, grauen, drehbaren Tisch ( $\varnothing 120$ cm) $1 \mathrm{~m}$ über dem Boden. Im Zentrum und je $30 \mathrm{~cm}$ vom Zentrum entfernt befanden sich Löcher; das Loch in der Mitte nahm während der Fütterung den Futternapf mit $2 \mathrm{~m}$ Zuckerwasser auf, in die beiden anderen Löcher wurden beim Test Attrappen von Futternäpfen ohne Zuckerwasser (ZW) eingesteckt. Als Lernsignal diente bei der Vordressur (s.u.) ein aus schwarzen Quadraten zusammengesetztes Kreuz (Abb. 1), bei der Dressur wurde es durch ein gleichgestaltetes gelbes Kreuz (Gelbkreuz) ersetzt. Die Alternative im kritischen Test war ein gleiehgestaltetes Blaukreuz. (Abb. 1 a-d). Die vertikale Anordnung bestand ebenfalls aus einer $1,20 \mathrm{~m}$ großen, runden, grauen Platte, die an einem Gerüst $1,50 \mathrm{~m}$ üher der Erde drehbar angeordnct 
war. Sie trug die gleichen Marken in der gleichen Größe, die - wie beim horizontalen Tisch mit dem Futtergefäß bzw mit den Attrappen durch ein Loch in ihrem Zentrum am Versuchstisch festgehalten wurden. Um jegliche Duftspuren auszuschalten, wurde bei beiden Anordnungen in der Testsituation ein $1,30 \mathrm{~m}(\varnothing)$ großes, ebenfalls graues Brett auf den Dressurtisch gelegt bzw davorgehängt.

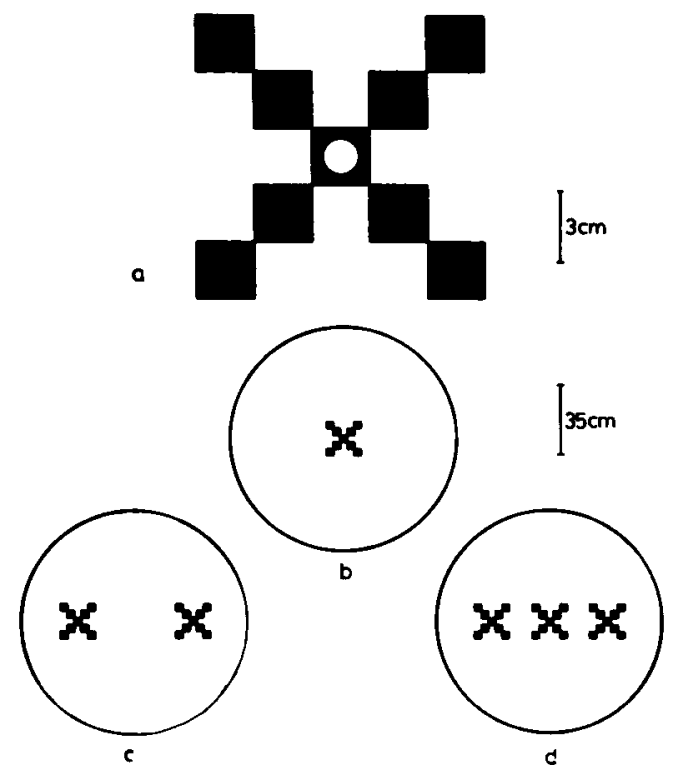

Aвв. 1. - a) Das Dressurmuster besteht aus 9 schwarzen bz. blauen bz. gelben Quadraten $(j e 2,5 \times$ $2,5 \mathrm{~cm}$ ), die ein aufgelöstes Kreuz bilden. Das mittlere Quadrat enthält ein Loch, in dem ein Plexiglasstempel steckt, der das Zuckerwasser enthält. Mit diesem Stempel wird die Marke am Tisch befestigt.

b) Anordnung der Marken auf der runden Dressurscheibe bei der Vordressur (schwarzes Kreuz) und bei den Lernakten (gelbes Kreuz);

c) Anordnung des Blau- und Gelbkreuzes in der Testsituation bei Versuchsserie I (2-Alternativen Situation);

d) Anordnung der Marken in der Testsituation bei Versuchsserie 2 (3-Alternativen Situation).

Fic. 1. - a) La figure servant au dressage se compose de 9 carrés $(2,5 \times 2,5 \mathrm{~cm})$ noirs, jaunes ou bleus dont l'assemblage forme une croix découpée. Le carré du milieu comporte un trou dans lequel est placé une pièce de plexiglas qui contient le sirop de sucre. Cette pièce de plexiglas sert à fixer la figure sur le panneau.

b) Disposition des figures sur le panneau circulaire pour le pré-dressage (croix noire) et pour l'acte d'apprentissage (croix jaune).

c) Disposition des croix bleue et jaune dans la situation de testage de la $1^{\text {re }}$ série d'essais (situation à 2 possibilités de choix).

d) Disposition des figures dans la situation de testage de la $2^{\mathrm{e}}$ série d'essais (situation à trois possibilités de choix).

\section{Versuchsablauf (2 Alternativen im Test)}

8-12 markierte Sammelbienen verkehrten am mittleren Schwarzkreuz und alarmierten Neulinge. Während des ersten Saugens eines Neulings auf dem schwarzen Kreuz wurde er als Versuchsbiene markiert und alle anderen Tiere weggefangen. Jetzt flog nur noch die eine Versuchsbiene (Vb). Diese wurde noch 2 mal auf dem Schwarzkreuz in der Mitte des Tisches belohnt (Vordressur). Nach dem 3. Abflug wurde die Testplatte mit den beiden Testmarken Gelbkreuz und Blaukreuz im gleichen Abstand von der Mitte auf den Dressurtisch gelegt. So fand die Vb beim 4. Anflug am früheren Dressurort (Mitte) keine Marke und keine Belohnung vor, aber 2 neue Marken im gleichen Abstand von der Mitte. Während des nun folgenden 4 Min langen Tests wurde das Suchverhalten dem zeitlichen Ablauf entsprechend protokolliert. 
Dabei wurde zwischen gezieltem Anfliegen einer der beiden Marken (A), dem Antippen einer Marke (T) und dem Setzen auf eine Marke (S) unterschieden (Menzel, 1967). Da die Vb bei der Vordressur auf keiner der beiden Marken belohnt worden war, bezeichnen wir diesen Test als “Spontanwahl ». Damit beim Test eine Ortsbevorzugung das Ergebnis nicht verfälscht, wurde jede Minute die relative Lage der beiden Testmarken durch Drehen der Tischplatte um $180^{\circ}$ verändert. Nach unserer Erfahrung ist dieses Drehen sehr wichtig, um zufällige Ortsbevorzugungen auszuschalten. Als bedeutsam hat sich auch herausgestellt, daß der Abstand zwischen den beiden Alternativen mindestens $50 \mathrm{~cm}$ beträgt (hier $60 \mathrm{~cm}$ ), da sonst gezielte Anflüge nicht mehr eindeutig angesprochen werden können.

Nach der Spontanwahl entfernten wir die Testplatte und boten der Vb in der Mitte des Tisches auf dem Gelbkreuz 2 m Zuckerwasser (1. Lernakt, LA). Nach der Rückkehr vom Stock wurde wiederum erst 4 Minuten lang geprüft $(\mathrm{Abb}$. $1 \mathrm{C})$ und dann erneut in der Mitte auf Gelbkreuz belohnt (2. LA). Nach 12 Tests wurde der Versuch meist abgebrochen. Auf diese Weise erhält man Lernkurven von einzelnen Vb (MenzeL, 1967); 12-15 Lernkurven bilden dann die im folgenden stets angegebene mittlere Lernkurve.

In einer 2. Versuchsserie (3 Alternativen) wurde nach einer gleichartigen Vordressur eine Spontanwahl über 2 Minuten in der oben beschriebenen Weise durchgeführt, in den folgenden 2 Minuten aber das Schwarzkreuz in der Mitte des Dressurtisches wieder geboten (Abb. 1d). In der anschließenden Dressur auf Gelbkreuz (wie oben beschrieben) wurde im Test jeweils das Schwarzkreuz in der Mitte des Versuchstisches zusätzlich aufgelegt und die Wahl dieser 3 Alternativen protokolliert. Auf diese Weise läßt sich der Umlernverlauf von Schwarzkreuz auf Gelbkreuz unter gleichen Bedingungen prüfen wie der Neulernverlauf auf Gelbkreuz.

Statistische Auswertung: Die gemittelte Wahlhäufigkeit nach jedem Lernakt der 12-15 Vb einer Serie wurde mit dem $\chi^{2}$-Test auf Homogenität geprüft; $p$ war stets größer 0,5 . Eine Streuungsgröße kann nicht angegeben werden, da die Vb pro Test unterschiedlich viele Entscheidungen trafen, der prozentuale Wert für die Richtigwahl für jede Vb daher unterschiedliches Gewicht hat. (In einem solchen Fall ist auch die Mittelung von Prozentwerten und die statistische Bearbeitung mit dem t-Test unzulässig). Die mittleren Lernkurven der verschiedenen Bienenrassen wurden mit dem Zeichentest von v.d. WAERDEN und NiEvergeLt (1956) auf ihre Unterschiede geprüft. Bei der zweiseitigen Anwendung dieses Tests wird festgestellt, mit welcher Irrtumswahrscheinlichkeit die Werte einer Kurve durchschnittlich größer oder kleiner sind als die Werte einer 2. Kurve. Für $p<0,01$ gilt der Unterschied als gut gesichert, für $p<0,05$ als schwach gesichert.

\section{ERGEBNISSE}

\section{1. - Versuchsserie : zwei Alternativen im Test}

\section{a. Lernverlauf und Wahlverhalten}

Lernen bedeutet Veränderung des Verhaltens mit dem Ziel einer besseren Anpassung an eine veränderte Umwelt. Der Lernvorgang ist ein Prozeß, der sich in der Zeit als Folge von Erfahrung abspielt. Je rascher sich das Tier auf eine neue Signalsituation einzustellen vermag, um so schneller arbeitet sein Lernsystem. Diesen Lernverlauf beschreibt die Lernkurve : die Veränderung der Wahrscheinlichkeit für das Auftreten einer Aktion als Funktion der Zahl belohnter gleichartiger Aktionen (Abb. 2-5).

Beobachtet man eine Biene beim Anflug auf eine Futterquelle, so läßt sich ihr Flugverhalten mit rasch wechselnden gezielten Anflügen, weiten und engen Schleifen, schwirrendem Stehen in der Luft und plötzlichem Zustoßen nur sehr schwer quantitativ beschreiben. Entscheidend ist daher die Verein- 
fachung der Prüfsituation (möglichst großer homogener Hintergrund, nur 2 Alternativen) und die Auswahl solcher Verhaltenskomponenten, die quantitativ ausgewertet werden können. Der ersten Forderung genügen unsere beiden Versuchsanordnungen (s. Methode), entsprechend der 2. Forderung unterscheiden wir in unserem Testprotokoll zwischen Anflug (A) : eine gezielte Zuwen-

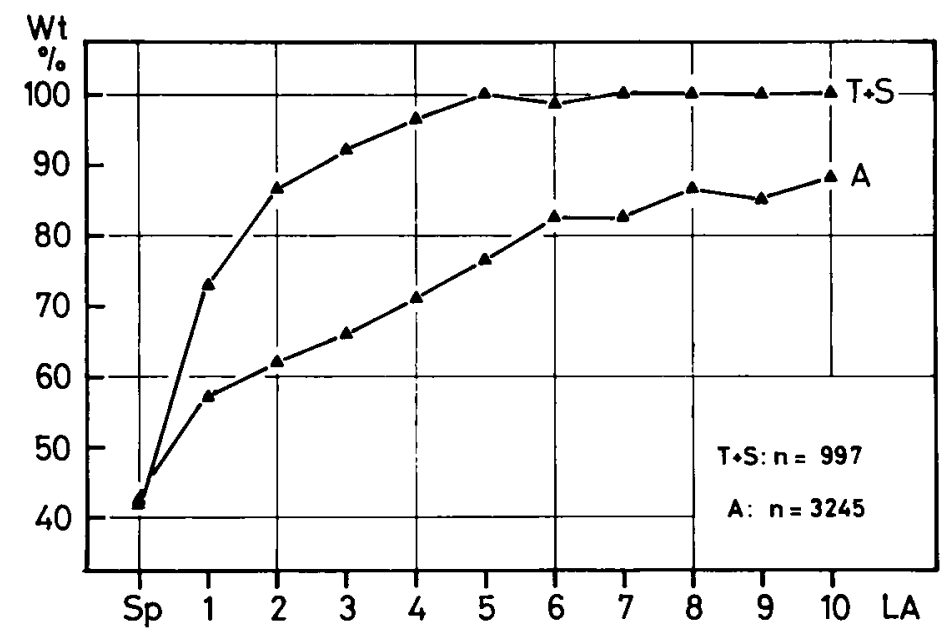

Aвв. 2. - Mittlere Lernkurven von A.m. carnica an der vertikalen Versuchsanordnung bei Auswertung der Anflugsentscheidungen $(A)$ und der Tipp-plus Sitzentscheidungen (T+S). Abszisse : Zahl der Lernakte auf Gelbkreuz (LA), Sp : Spontanwahl; Ordinate : Wahltendenz (Wt) in \% der Richtigwahlen für Gelbkreuz. $n$ gibt die Zahl der Entscheidungen an.

Fig. 2. - Courbes d'apprentissage moyennes d'A.m. carnica dans le dispositif vertical, obtenues à partir des décisions d'approche $(A)$ et des décisions de contact et d'atterrissage $(T+S)$.

Abscisses : nombre des actes d'apprentissage sur la croix jaune (LA); Sp choix spontané. Ordonnées : tendance du choix $(\mathrm{Wt})$ en $\%$ des choix corrects pour la croix jaune. n : nombre de décisions.

dung zur Testmarke aus mindestens $15 \mathrm{~cm}$ Entfernung verbunden mit einer verringerten Fluggeschwindigkeit über der Marke, Antippen (T) : einem Anflug, der bis zur Berührung der Marke mit den Beinen oder/und den Fühlern führt, und dem Hinsetzen auf der Marke (S), wobei die Flügel mindestens $1 \mathrm{sec}$ eingefaltet sind. Dies ist nicht eine formale Einteilung des Verhaltens, sondern spiegelt die hierarchische Gliederung der Wahlaktionen wieder. Die Gesamtheit $\operatorname{der}$ A, T und S- Entscheidungen sowie ihr Verhältnis (u.z. der Zahl der A zu der der $\mathbf{T}+$ S-Entscheidungen) wird im folgenden mit « Suchstrategie » der $\mathrm{Vb}$ bezeichnet.

Ein Ausdruck dieser Verhaltensstruktur ist, daß die Lernkurven beschrieben mit den A-, T- und S-Entscheidungen unterschiedlichen Verlauf haben. (Abb. 2). Wertet man die $S$ und T-Entscheidungen aus, erhält man steilere Lernkurven als bei Auswertung der A-Entscheidungen. Von den insgesamt 
31026 protokollierten Anflügen führte nur ein viertel (7075) zu T- oder SEntscheidungen. - Antippen und Setzen auf einer Marke wird also präziser gesteuert als Anfliegen. Dies gilt für alle 4 Rassen.

Für die Interpretation der Lernkurven bei der Auswertung der Anflüge hat dies eine wichtige Folge : Unterscheiden sich z.B. 2 Rassen im Verhältnis der $A$ - und $T+S-E n t s c h e i d u n g e n$, so führt das zu einer Veränderung der Lernkurve : relativ mehr T- und S-Entscheidungen haben eine steilere Lernkurve zur Folge. Weiterhin : ändert sich im Verlauf des Lernverhaltens die Suchstrategie derart, daß relativ mehr T- und S-Entscheidungen auftreten, muß sich allein aufgrund dieser Verhältnisänderung eine bessere Wahl der Lernmarke ergeben. Es ist auch nicht auszuschließen, daß sich das Verhältnis der prozentualen Richtigwahl (Wahltendenz) bei A- und $T$ + S-Entscheidungen im Lernverlauf oder an verschiedenen Versuchsanordnungen ändert; in diesem Fall sprechen wir von einer Veränderung der Kopplung zwischen A- und $\mathbf{T}+$ S-Wahltendenz. Es gilt daher bei unterschiedlichen Lernkurven zu prüfen, ob wirklich Lernunterschiede vorliegen, und welche Rolle etwa eine veränderte Wahlstrategie oder/und eine andere Kopplung zwischen $A$ - und $\mathbf{T}+$ S-Wahltendenz spielen.

Bienen zeigen gegenüber Testfarben und -formen ein unterschiedliches spontanes Wahlverhalten (Hertz, 1931, Menzed, 1967), das durch die individuelle Vorgeschichte der $\mathrm{Vb}$ oder durch eine rassenspezifisch unterschiedliche Attraktivität der beiden Testmarken bedingt sein könnte. Um den eigentlichen Lernvorgang von diesen recht unspezifischen Faktoren abzuheben, ist es entscheidend wichtig, das Wahlverhalten und die Wahltendenz jeder einzelnen $\mathrm{Vb}$ vor Lernbeginn, also vor der 1 . Belohnung auf der Lernmarke zu kennen. Ohne Kenntnis des Wahlverhaltens vor Lernbeginn könnten Unterschiede als Lernunterschiede interpretiert werden, die z.B. auf einen rassenspezifischen Unterschied in der Attraktivität der Testmarken zurückgehen.

Um Wahlen vor der Konditionierung auf eine Testmarke zu erhalten, werden die Versuchstiere zuerst auf einem solchen Signal belohnt, das beiden späteren Alternativen ähnlich ist, hier also die Form des Kreuzes. Gegenüber dem dann zu lernenden neuen Parameter - die Farbe des Kreuzes - handelt es sich im 1. Test um eine " Spontanwahl ». Da das gelbe Kreuz aufgrund der geringeren Attraktivität der gelben Farbe etwas schwächer als das Blaukreuz gewählt wird (Abb. 3 und 4), dressierten wir anschließend auf das Gelbkreuz.

In einem 4 Minuten langen Test nach jeder Belohnung auf Gelbkreuz erhält man im Mittel 21,5 A-Entscheidungen und 3,9 T + S-Entscheidungen (über rassenspezifische Unterschiede siehe unten). Die Vermutung, daß so viele unbelohnte Wahlen zu einer Abdressur und damit zu einer schlechteren Wahl des Gelbkreuzes im Verlauf eines Tests führen könnten, bestätigt sich 
R. MENZEL, H. FREUDEL, U. RÜHL

nicht : bei keiner Rasse nimmt die Richtigwahl im Verlauf des Tests systematisch ab; die Unterschiede in der mittleren Wahl getrennt für jede Minute ausgewertet betragen maximal $6 \%$. Häufig wählen die Vb in der 3. und 4 . Minute besser als zu Beginn des Tests (vgl. MenzeL, 1967).

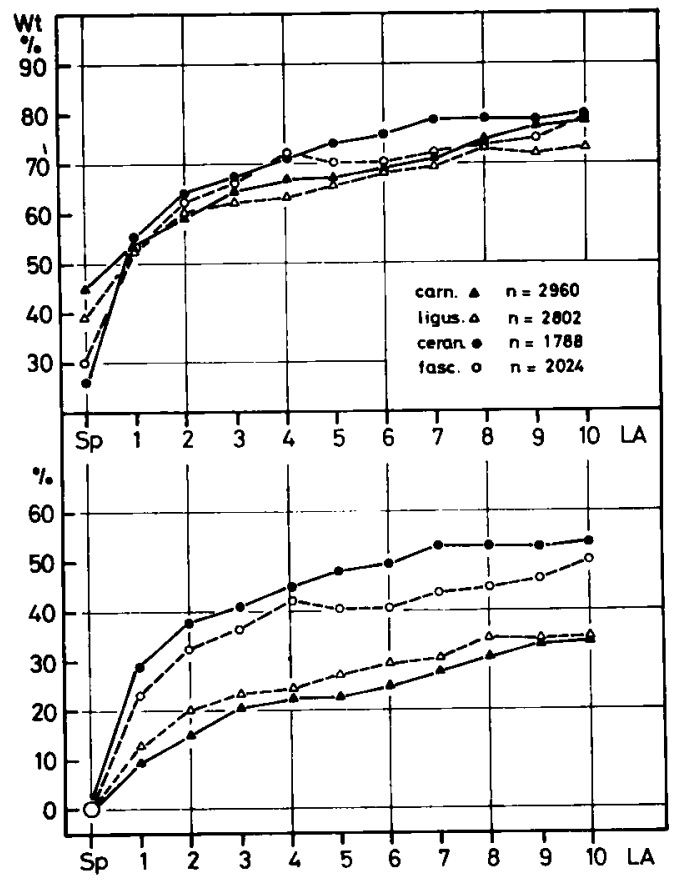

Aвв. 3. - Mittlere Lernkurven der 4 Rassen an der horizontalen Versuchsanordnung bei Auswertung der Anflugsentscheidungen.

-A一 : A.m. carnica,

$-\Delta-A . m$. ligustica,

- A. cerana,
$-\mathrm{O}$ - A.m. lamarckii (früher A.m. fasciata)

Abszisse : Zahl der Lernakte (LA) auf Gelbkreuz; Ordinate obere Abb. : Wahltendenz (Wt) in \% der Entscheidungen für Gelbkreuz, untere Abb. : Prozentuale Änderung der Wahlen für Gelbkreuz bezogen auf die mittlere Spontanwahl jeder Rasse (Lernzuwachskurve). n gibt die Zahl der A-Entscheidungen wieder.

Fig. 3. - Courbes d'apprentissage moyennes des 4 races dans le dispositif horizontal obtenues à partir des décisions d'approche.

$-\Delta-=$ A.m. carnica

$-\Delta-=A . m$. ligustica

$-\mathrm{O}=$ A. cerana
$-\mathrm{O}=$ A.m. lamarckii

Abscisses : nombre des actes d'apprentissage (LA) sur croix jaune.

Ordonnées : figure du haut : tendance de choix $(W t)$ en $\%$ des décisions en faveur de la croix jaune. Figure du bas : pourcentage de modification des choix pour la croix jaune par rapport au choix spontané moyen de chaque race (courbe de croissance de l'apprentissage), $n$ : nombre des décisions A. 


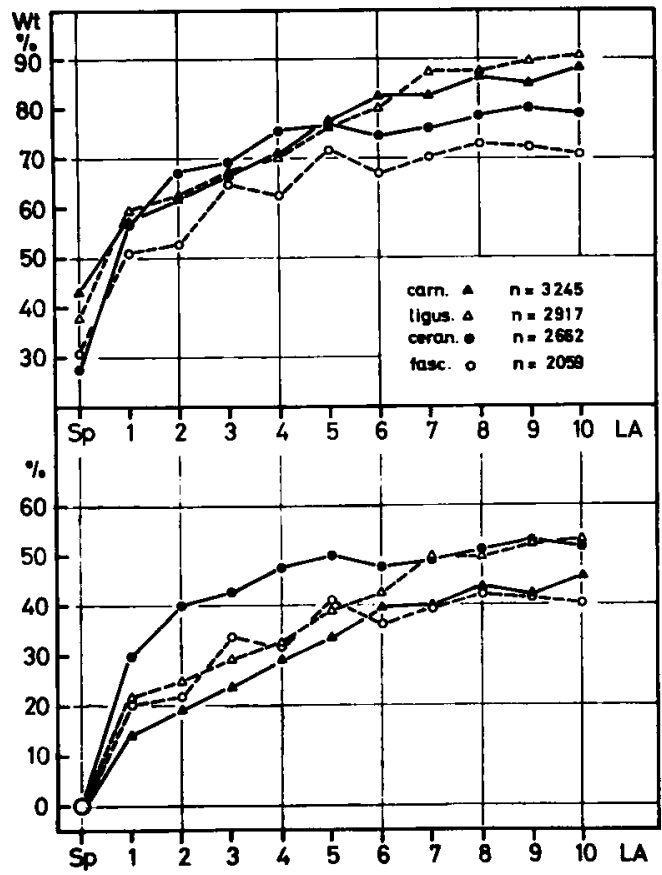

Aвв. 4. - Mittlere Lernkurven der 4 Rassen an der vertikalen Versuchsanordnung bei Ausvertung der Anflugsentscheidungen (vgl. Abb. 3).

FIG. 4. - Courbes d'apprentissage moyennes des 4 races dans le dispositif vertical obtenues d̀ partir des décisions d'approche (comparer avec fig. 3).

\section{b. Rassenspezifische Lernunterschiede}

Wie die Abb. 3a und 4a zeigen, wählen die 4 Bienenrassen die Alternativen auf beiden Versuchsapparaturen spontan unterschiedlich : A.m. carnica zeigt die am besten ausgeglichene Wahl (45\% bzw $43 \%$ für Gelbkreuz). Auf der horizontalen Anordnung hat A.m. lamarckii (32\%) und auf der vertikalen A. cerana $(27,4 \%)$ die geringste Wahltendenz gegenüber dem Gelbkreuz. Der anschließende Lernverlauf weist für die einzelnen Rassen unterschiedliche Steilheit auf. Bei horizontal (Abb 3a) und vertikal (Abb 4a) angeordneten Marken haben die indischen Bienen die steilste Lernkurve, die Kärntnerbienen dagegen eine viel flachere.

Die rassenspezifischen Unterschiede werden aber durch die unterschiedliche Ausgangsbevorzugung verdeckt. Es ist deshalb günstiger, nicht die tatsächliche prozentuale Wahltendenz aufzutragen, sondern die Kurven auf ihren jeweiligen Ausgangswert zu beziehen und den prozentualen Lernzuwachs in Abbängigkeit von der Zahl der Lernakte anzugeben (Abb. 3b, 4b). 
R. MENZEL, H. FREUDEL, U. RÜHL

Dann wird ganz klar, daß an beiden Versuchsanordnungen die indischen Bienen am raschesten das Gelbkreuz lernen. (In Abb. 3b ist ihre Lernkurve von der von A.m. carnica und A.m. ligustica signifikant verschieden, in Abb. 4h von $A$.m. carnica und $A$.m. lamarckii, von $A$.m. ligustica nur bis zum 5. LA). Auf der horizontalen Anordnung ist die Lernkurve der ägyptischen Biene sehr steil und damit dem Lernverlauf der $A$. cerana ähnlich; demgegenüber lernen die Italiener- und Kärntner Bienen das Gelbkreuz langsamer und wählen auf dem Plateau der Lernkurve weniger sicher. Auf der vertikalen Anordnung (Abb. 4b) zeigen A.m. ligustica, carnica und lamarckii während der ersten 6 Lernakte etwa den gleichen Lernverlauf. Carnica und lamarckii erreichen dann ihr Lernniveau, während ligustica weiter die Wahl verbessert.

Da der Verlauf der Lernkurve, wie oben gezeigt wurde, auch von der Suchstrategie des Versuchstieres abhängt, muß als nächstes geprüft werden, ob solche Unterschiede bei den vier Rassen vorliegen. Es wäre auch denkbar, daß sich das Wahlverhalten der Vb an der horizontalen und vertikalen Anordnung unterscheidet und die steileren Lernkurven aller Rassen vertikalen Marken gegenüber darauf zurückführen lassen.

Wie aus einem Vergleich der globalen Suchstrategie (Tab. 1) hervorgeht, ist die Zahl der $\mathbf{T}+\mathrm{S}$-Entscheidungen an der vertikalen Anordnung relativ

TAB. 1 .

TABL. 1 .

\begin{tabular}{|c|c|c|c|c|c|c|}
\hline & \multicolumn{3}{|c|}{$\begin{array}{c}\text { Horizontale Versuchsanordnung } \\
\text { Dispositif horizontal }\end{array}$} & \multicolumn{3}{|c|}{$\begin{array}{c}\text { Vertikale Versuchsanordnung } \\
\text { Dispositif vertical }\end{array}$} \\
\hline & A & $\mathbf{T}+\mathbf{S}$ & $\frac{\mathbf{A}}{\mathbf{T}+\mathrm{S}}$ & $\mathbf{A}$ & $\mathbf{T}+\mathbf{S}$ & $\frac{A}{T+S}$ \\
\hline cerana $\ldots \ldots \ldots \ldots \ldots \ldots \ldots$ & 1788 & 418 & 4,28 & 2662 & 794 & 3,36 \\
\hline lamarckii $\ldots \ldots \ldots \ldots \ldots \ldots$ & 2024 & 470 & 4,31 & 2059 & $\mathbf{5 5 0}$ & 3,74 \\
\hline carnica..................... & 2960 & 579 & 5,11 & 3245 & 997 & 3,26 \\
\hline ligustica $\ldots \ldots \ldots \ldots \ldots \ldots$ & 2802 & 548 & 5,11 & 2917 & 1046 & 2,79 \\
\hline
\end{tabular}

größer als auf der horizontalen. Dies wird besonders deutlich bei den Rassen carnica und ligustica (das Verhältnis $\frac{A}{T+S}$ ist auf der vertikalen um den Faktor 1,58 bzw 1,84 kleiner als auf der horizontalen), während vor allem cerana, aber auch lamarckii an beiden Anordnungen ein ähnliches Wahlverhalten zeigen. Tatsächlich verlaufen die Lernkurven von carnica und ligustica auf dem horizontalen Dressurtisch signifikant flacher als auf dem vertikalen, während keine Unterschiede in den Lernkurven von cerana auftreten. Auch 
bei A.m. lamarckii sind die Unterschiede der Lernkurven an beiden Anordnungen nicht signifikant; es fällt aber auf, daß bei vertikalen Marken nach den ersten 4 LA die Wahlsicherheit geringer ist (Abb. 3 und 4), was nach dem Verhältnis der $\mathbf{A}$ und $\mathbf{T}+\mathrm{S}$-Entscheidungen nicht zu erwarten wäre.

Dieser summarische Vergleich der Häufigkeit von A und T + S-Entscheidungen in den beiden Testsituationen kann nur ein sehr grober Anhaltspunkt für den Einfluß der Suchstrategie auf den Lernverlauf der Lernkurven sein, da sich im Lernverlauf die Suchstrategie verändern könnte. Dies wurde für die Daten, die an der vertikalen Versuchsanordnung gewonnen wurden, geprüft und trifft, wie Abb. 5 zeigt, zu : Bei A.m. carnica und ligustica nimmt die Zahl der T + S-Entscheidungen im Lernverlauf relativ stärker ab als die der A-Entscheidungen (das Verhältnis $\frac{\mathrm{A}}{\mathrm{T}+\mathrm{S}}$ wächst von 2,58 auf 3,36 bzw. 2,72 auf 3,12). Bei A.m. lamarckii und $A$. cerana dagegen bleibt die Zahl der AEntscheidungen im Lernverlauf etwa gleich, bei A.m. lamarckii auch die der $\mathrm{S}+\mathrm{T}$-Entscheidungen, bei $A$. cerana dagegen nimmt die Zahl der $\mathrm{T}+\mathrm{S}$ Entscheidungen zu. Die Suchstrategie ändert sich bei $A$. cerana im Lernverlauf also derart, daß sicherere Entscheidungen relativ häufiger auftreten, bei $A$.m. carnica und ligustica relativ weniger häufig auftreten und bei $A . m$. lamarckii etwa gleich bleiben. Das hohe Lernineau der indischen Biene wird demnach auch durch die Reduktion unsicherer und Zunahme sicherer Entscheidungen erreicht. Der weitere Anstieg der Lernkurve von A.m. ligustica an der vertikalen Anordnung (Abb. 4b), der bis zu dem hohen Lernniveau der indischen Biene führt, läßt sich mit einer Veränderung der Suchstrategie nicht erklären.

Der Lernverlauf wäre aber nur dann hinreichend mit der Anflug-Lernkurve (Abb. 3 und 4) und der Suchstrategie beschrieben, wenn man annehmen könnte, daß die Kopplung zwischen A-Wahltendenz und T + S-Wahltendenz bei allen Rassen gleich und von Versuchsanordnung sowie Lernverlauf unabhängig wäre (siehe oben). Ein Vergleich aller Lernkurven nach A- und T + SEntscheidungen zeigt aber, daß diese Annahme nicht zutrifft. Da eine Analyse auf rassenspezifische Unterschiede verweist, soll im folgenden darauf eingegangen werden. Der besseren Übersicht halber werden nicht alle Lernkurven abgebildet, sondern nur für jede Rasse der Quotient aus $\frac{\mathrm{A} \%}{\mathrm{~T}+\mathrm{S} \%}$ (wobei $\%$ bedeuten soll : prozentuale Richtigwahl nach Auswertung der A bzw T + SEntscheidungen).

Da sich in der Spontanwahl nach allen 3 Verhaltenskategorien ein etwa gleicher Prozentsatz der Wahltendenz ergibt, daran anschließend in jedem Fall die Lernkurve nach $\mathbf{T}+\mathbf{S}$-Entscheidungen steiler ansteigt als nach $\mathbf{A}$ Entscheidungen, folgt bei allen Versuchstieren in der ersten Lernphase ein 
starker Abfall des genannten Quotienten (Abb. 6). Dieser Abfall wird umso größer sein, je mehr die T + S-Lernkurve die A-Lernkurve an Steilheit übertrifft (Abb. 6 a : ligustica, carnica, lamarckii; $6 \mathrm{~b}$ : cerana, lamarckii, carnica). Wenn sich bei fortschreitendem Lernen die A-Lernkurve dem Niveau der S-Lernkurve nähert, wird der Quotient $\frac{\mathrm{A} \%}{\mathrm{~T}+\mathrm{S} \%}$ wieder dem Wert 1 zustreben (Abb. 6a cerana); bleibt das Lernniveau der A-Lernkurve aber immer unter dem der S-Lernkurve, wird die Quotientenkurve einen entsprechenden mittleren Wert angeben.

Wie die Abb. 6a und 6b zeigen, ergeben sich nicht nur Unterschiede im Verlauf der A- bzw T + S-Lernkurven zwischen den 4 Rassen, sondern auch bei einer Rasse an den beiden Versuchsanordnungen : 1.) Bei $A$. cerana ist auf der horizontalen Anordnung auch die A-Lernkurve sehr steil und erreicht nahezu das gleiche Niveau wie die $\mathrm{T}+\mathrm{S}$-Lernkurve; auf der vertikalen Anordnung dagegen verläuft die A-Lernkurve mit geringerer Steigung und niedrigerem Niveau als die $\mathrm{T}+\mathrm{S}$-Lernkurve. 2.) Bei A.m. ligustica sind die Verhältnisse gerade umgekehrt; den vertikalen Marken gegenüber wird im Lernverlauf die A-Entscheidung so sicher wie die $\mathbf{T}+$ S-Entscheidung. Tatsächlich steigt nur bei A.m. ligustica die A-Lernkurve auch nach dem 5. LA noch weiter an (Abb. 4a) und erreicht das Niveau von $90 \%$ richtige A-Wahlen. 3.) Die T + S-Lernkurve von $A$.m. carnica verläuft an der vertikalen Anordnung steiler als an der horizontalen. Da die A-Lernkurve nur bei vertikalen Marken sich stärker der S-Lernkurve nähert (Abb. 6b), erreicht die Kärntner Biene an der vertikalen Anordnung auch in den A-Entscheidungen eine höhere Sicherheit. 4.) Beim Vergleich der Reihenfolge in den A-Lernkurven der 4 Rassen an den beiden Versuchsanordnungen (Abb. 3 und 4) ergab sich vor allem bei A.m. lamarckii eine auffallende Abweichung : Während sie horizontale Marken ähnlich rasch und gut lernte wie $A$. cerana, stieg ihre Lernkurve bei vertikalen Marken nicht im gleichen Maße an wie bei den anderen Rassen, was zu einer relativ flacheren und niedrigeren Lernkurve führte. An Hand der Abb. 6 läßt sich nun zeigen, daß dieser Unterschied auf einer veränderten Kopplung zwischen $\mathrm{A}$ - und $\mathrm{T}+\mathrm{S}-\mathrm{Wahltendenz}$ an den beiden Versuchsanordnungen beruht : während bei $A$.m. carnica und ligustica die A-Lernkurven bei vertikalen Marken sich den $\mathrm{T}+\mathrm{S}$-Lernkurven stärker nähern, wird der Unterschied zwischen diesen beiden Lernkurven bei A.m. lamarckii größer. Tatsächlich ordnet sich die ägyptische Biene auch an der vertikalen Versuchsanordnung in ihrer Lerngeschwindigkeit und dem Lernniveau der indischen Biene zu, wenn man die $\mathrm{T}+\mathrm{S}$-Lernkurven betrachtet (Abb. 6).

Eine Verbesserung der A-Wahlsicherheit (Abb. 3 und 4) kann nach der bisherigen Analyse grundsätzlich auf 3 Faktoren beruhen : 1. der zunehmenden prozentualen A-Wahltendenz, 2. dem häufigeren Auftreten von $\mathbf{T}+\mathrm{S}$ - 
Entscheidungen im Verhältnis zu den A-Entscheidungen (Verkleinerung des Quotienten $\left.\frac{A}{\mathrm{~T}+\mathrm{S}}\right)$ und 3. einer Veränderung der Kopplung zwischen AWahltendenz und $\mathbf{T}+\mathrm{S}-\mathrm{Wahltendenz}$ in Richtung einer Vergrößerung des Quotienten $\frac{\mathrm{A} \%}{\mathrm{~T}+\mathrm{S} \%}$ (s.o. und Abb 6). Der entscheidende Faktor für die zunehmende Wahl der belohnten Marke ist sicherlich der unter 1. genannte. Der Vergleich zwischen den 4 Rassen und den beiden Versuchsanordnungen zeigt aber, daß auch die beiden anderen Faktoren von großer Bedeutung sind.

A. cerana weist unter allen Gesichtspunkten das rascheste Lernvermögen auf; sie verändert ihre Suchstrategie im Lernverlauf so, daß sicherere Entscheidungen häufiger werden. Die Kopplung zwischen A-Wahltendenz und $\mathbf{T}+\mathbf{S}$ Wahltendenz an den beiden Versuchsanordnungen ist zwar unterschiedlich $\left(\frac{\mathrm{A} \%}{\mathrm{~T}+\mathrm{S} \%}\right.$ horizontal größer als vertikal, vgl. Abb. 6$)$, dies wirkt sich aber auf die Lernkurven aufgrund ihres sehr steilen Anstiegs nur geringfügig aus.

A.m. lamarckii kommt dem Lernverhalten der indischen Biene am nächsten. Auch sie verändert - wenn auch geringfügiger - bei zunehmendem Lernfortschritt ihre Suchstrategie in Richtung eines häufigeren Auftretens von $\mathrm{T}+\mathrm{S}$-Entscheidungen. Der flachere Verlauf der A-Lernkurve von vertikalen Marken läßt sich auf eine Veränderung der Kopplung zwischen A-Wahltendenz und $\mathbf{T}+\mathbf{S}-$ Wahltendenz $\left(\right.$ Verkleinerung des Quotienten $\left.\frac{\mathbf{A} \%}{\mathbf{T}+\mathbf{S} \%}\right)$ zurückführen.

Die Rassen A.m. carnica und ligustica weisen flachere A-Lernkurven auf als die beiden anderen Rassen. Sie verbessern ihre Suchstrategie im Lernverlauf nicht, sondern verschlechtern sie geringfügig (Abb. 5). Bei beiden Rassen wird besonders deutlich, daß sich die Kopplung zwischen A-Wahltendenz und $\mathrm{T}+\mathrm{S}-\mathrm{Wahltendenz}$ an den beiden Versuchsanordnungen so verändert, daß der Quotient $\frac{\mathrm{A} \%}{\mathrm{~T}+\mathrm{S} \%}$ vertikalen Marken gegenüber größer wird. Das hat zur Folge, daß (vor allem bei $A$.m. ligustica) das vertikale Gelbkreuz nur anfänglich schlechter gelernt wird als von der indischen Biene.

\section{c. Rassenspezifische Aktivitätsunterschiede}

Die Dressuren der 4 Bienenrassen konnten nicht gleichzeitig durchgeführt werden. Sie erstreckten sich über mehrere Sommermonate in 2 Jahren (1970 und 1971). Während dieser Zeit ändern sich eine ganze Reihe von Faktoren, die den Lernvorgang beeinflussen könnten : Temperatur, Beleuch- 
tungsverhältnisse, Nektarangebot in der Umgebung und damit möglicherweise der subjektive Belohnungswert einer $2 \mathrm{~m}$ Zuckerlösung, Nahrungsbedarf des Volkes, Brutverhältnisse, Alter der Versuchstiere und vieles mehr. Eine Temperaturabhängigkeit der Lernkurven ließ sich wie schon in früheren Experimenten (MenzeL, 1967, 1968, 1969) nicht finden. Die wechselnden Beleuchtungsverhältnisse durch Bewölkung oder im Lauf des Tages haben wir nicht systematisch registriert; ein Zusammenhang mit der Steilheit der Lernkurven
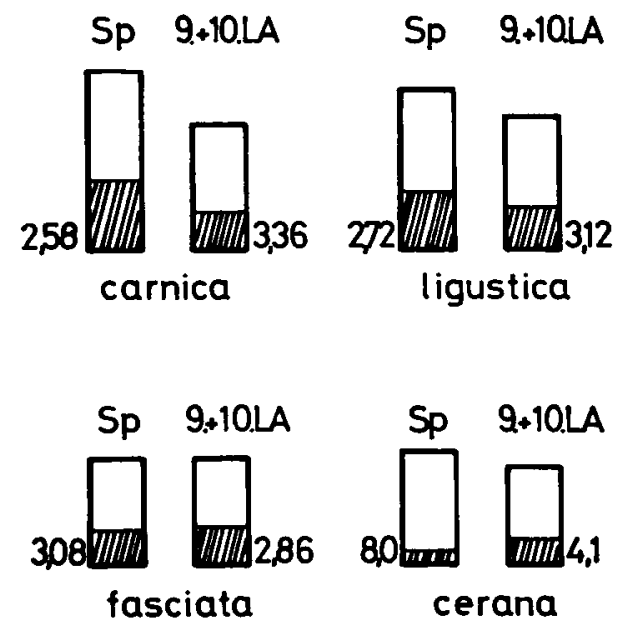

Авв. 5. - Anderung der Suchstrategie der 4 Rassen an der vertikalen Versuchsanordnung.

Jede Säule gibt die mittlere Zahl der Anflugsentscheidungen im 4-Minuten-Test an, der schräg schraffierte Teil der Säule die Anzahl der T + S-Entscheidungen. Unter Sp findet man die Verhältnisse bei der Spontanwahl, unter 9. + 10. LA die während des Tests nach dem 9. und 10. LA. Die Zahl neben jeder Säule bezeichnet den Quotienten aus

$$
\frac{\text { A-Entscheidungen }}{\mathbf{T}+\text { S-Entscheidungen }} \text { (siehe Text). }
$$

FIG. 5. - Modification de la stratégie exploratrice des 4 races dans le dispositif vertical.

Chaque colonne donne le nombre moyen de décisions d'approche dans un test de 4 minutes. La partie hachurée des colonnes dónne le nombre des décisions $T+S$. Sous Sp on trouve les proportions dans le choix spontané. Sous $9+10 \mathrm{LA}$ la proportion pendant les tests 9 et 10 LA. Le nombre à côté de chaque colonne donne le quotient

$$
\frac{\text { décisions } A}{\text { décisions } T+S}
$$

fiel uns aber bei keiner der 4 Rassen auf. Sehr viel schwieriger ist eine Kontrolle, welche Rolle ein möglicher Wechsel im Belohnungswert der konstant mit gleicher Konzentration gebotenen Zuckerlösung spielt. Von A.m. carnica wissen wir, daß eine Veränderung der Zuckerwasserkonzentration oder der Belohnungsmenge über einen großen Bereich ohne Ein $\mathcal{A} ß$ auf den anfänglichen Lernverlauf ist (Menzel und Erber, 1972). Das muß für alle Rassen gelten. Wir prüften diese Frage deshalb für A. cerana.

Die Abb. 8 zeigt, daß sich für 2 verschiedene Belohnungsarten $(2 \mathrm{~m}$ Zuckerwasser, Honigwasser) die gleiche steile Lernkurve ergibt. Wir vermuten, 
daß die für A.m. carnica und A. cerana gefundene weitgehende Unabhängigkeit der Lernkurve von der Belohnungsart auch für die beiden anderen Rassen gilt.

Dressiert man Honigbienen zu verschiedenen Jahreszeiten, dann beobachtet man, daß die Sammelaktivität an einer künstlichen Futterquelle nicht nur vom Wetter sondern sehr stark vom natürlichen Nektarangebot in der Umge-

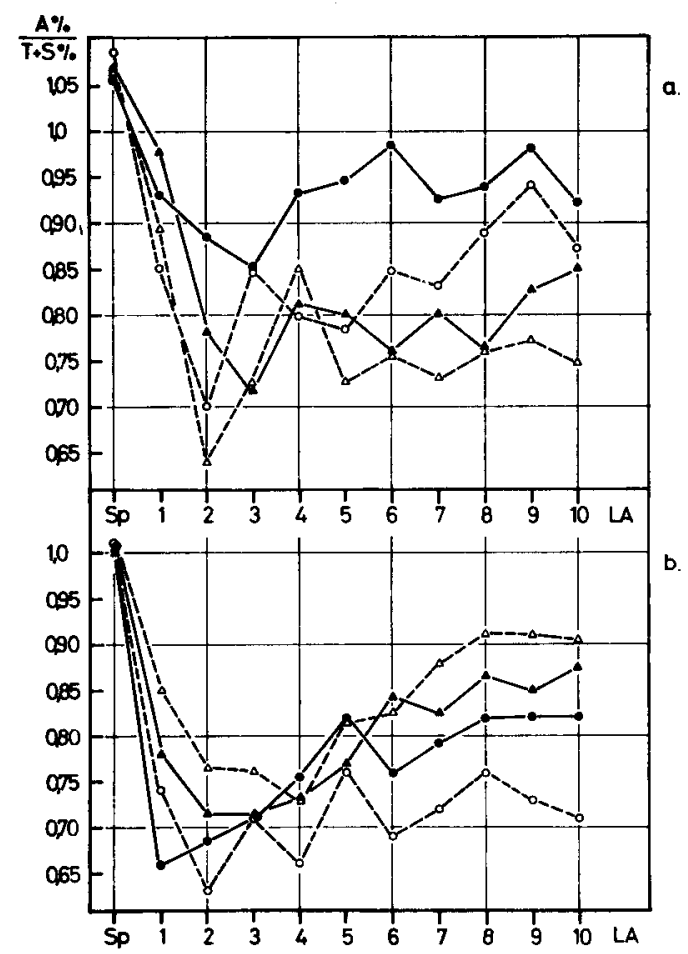

Авв. 6. - Vergleich der mittleren Lernkurven bei Auswertung der Anflugs- (A) und Tipp-plus Sitz- $(T+S)$ - Entscheidungen für jede der 4 Rassen an der horizontalen (a) und vertikalen (b) Versuchsanordnung.

Abszisse : Zahl der Lernakte (LA), Sp : Spontanwahl;

Ordinate: Quotient aus der prozentualen Richtigwahl bei A und $\mathbf{T}+$ S-Entscheidungen $\left(\frac{\mathrm{A} \%}{\mathrm{~T}+\mathrm{S} \%}\right)$, vergl. Text.

$-\mathbf{A}-$ A. m. carnica, $-\triangle-$ Q. m. ligustica, $-\mathbf{-}-$ A. cerana, $-\mathrm{O}-$ A. m. lamarckii (früher A. m. fasciata).

FIG. 6. - Comparaison des courbes d'apprentissage moyennes obtenues à partir des décisions d'approche $(A)$ et des décisions de contact et d'atterrissage $(T+S)$ pour chacune des races dans le dispositif horizontal (a) et vertical (b).

Abscisses : nombre des actes d'apprentissage (LA). Sp, choix spontané.

Ordonnées : quotient des pourcentages de choix corrects dans les décisions A et dans les décisions $\mathrm{T}+\mathrm{S}\left(\frac{\mathrm{A} \%}{\mathrm{~T}+\mathrm{S} \%}\right)$.

$-\mathbf{A}-=$ A. m. carnica $-\triangle-=$ A. m. ligustica $-\mathbf{O}=$ A. cerana $-\mathrm{O}-=$ A. m. lamarckii. 
bung abhängt. Im Herbst erhöht sich die Sammelfrequenz und die Ausdauer, mit der nach einer versiegten Futterquelle gesucht wird. Diese Sammelmotivation (quantifiziert in Anflügen pro Testminute, A/Min) könnte ein Indikator für eine wechselnde Einstellung zum Futtereintragen sein.

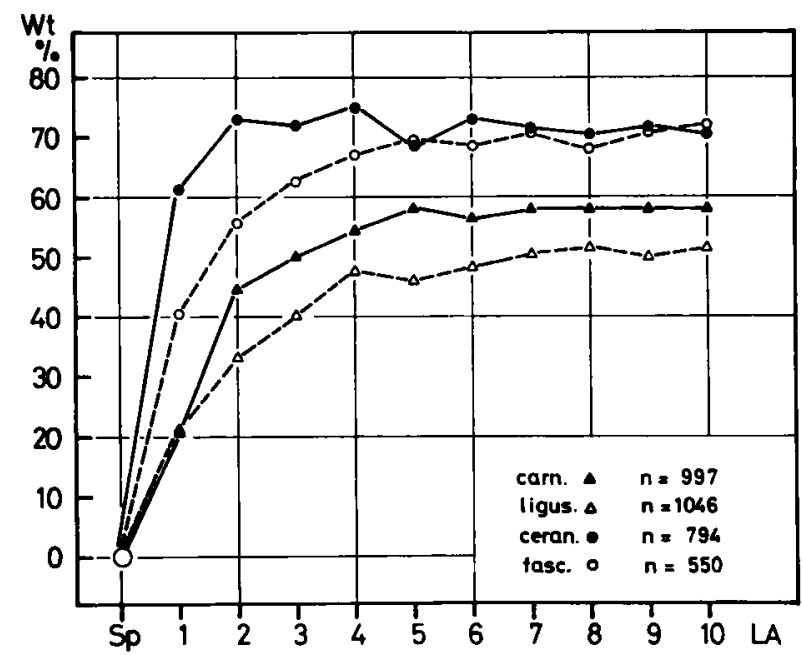

Авв. 7 - Mittlere Lernkurven der 4 Rassen an der vertikalen Versuchsanordnung bei Auswertung der Tipp- und Sitzentscheidungen $(T+S)$.

Abzsisse : Zahl der Lernakte (LA), Spontanwahl;

Ordinate : Prozentuale Änderung der Wahltendenz für Gelbkreuz bezogen auf die mittlere Spontanwahl jeder Rasse (Lernzuwachskurve). $n$ gibt die Zahl der $\mathbf{T}+\mathrm{S}$-Entscheidungen an $-\Delta-A$. m. carnica, $-\triangle-A$. m. ligustica, $-\mathbf{- A}$. cerana, $-\mathrm{O}-\mathbf{A}$. m. lamarckii (früher A. m. fasciata).

Fig. 7. - Courbes d'apprentissage moyennes des 4 races dans le dispositif expérimental vertical, obtenues à partir des décisions de contact et d'atterrissage $(T+S)$.

Abscisses : nombre des actes d'apprentissage (LA). Sp, choix spontané.

Ordonnées : pourcentage de modifications de la tendance de choix en faveur de la croix jaune rapporté au choix spontané moyen de chaque race (courbe de croissance de l'apprentissage). n. nombre des décisions $T+S \cdot-\Delta-=A$. m. carnica $-\triangle-=A$. m. ligustica $-\mathbf{A}$. cerana $-\mathrm{O}-=$ A. m. lamarckii.

In früheren Experimenten (Menzel 1967, 1968) fanden wir, daß bei A.m. carnica kein Zusammenhang zwischen Sammelmotivation und Lernkurve bzw Erinnerungsvermögen im langzeitigen und kurzzeitigen Bereich besteht. Dies bestätigt sich auch bei diesen Versuchen, wenn man die Vb der gleichen Rasse untereinander vergleicht : eine höhere Zahl von Anflügen im Test oder ein rascheres Zurückkehren vom Stock nach der Belohnung läßt sich nicht mit einem besseren oder schlechteren Lernverlauf korrellieren. Da sich die 4 geprüften Rassen in ihrer Sammelmotivation und ihrer Suchstrategie (vgl. Abb. 6 und Tab. 1) unterscheiden, bleibt zu prüfen, ob sich beim Vergleich der verschiedenen Rassen ein Zusammenhang zwischen der Aktivität an der Futterstelle und dem Lernverlauf feststellen läßt. 


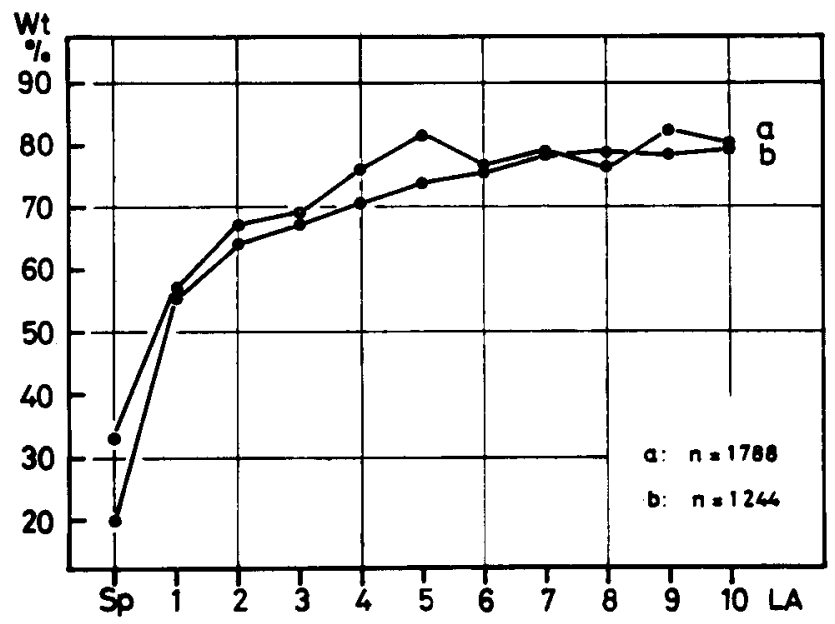

Aвв. 8. - Mittlere Lernkurven von A. cerana an der horizontalen Versuchsanordnung bei Auswertung der Anfugsentscheidungen.

a) Belohnung mit $2 \mathrm{~m}$ Zuckerwasser, b) Belohnung mit Honigwasser. Abszisse : Zahl der Lernakte (LA), Sp : Spontanwahl Ordinate : prozentuale Wahl des Gelbkreuses (Wt.). n gibt die Zahl der A-Entscheidungen an.

FIG. 8. - Courbes d'apprentissage moyennes d'A. cerana dans le dispositif expérimental horizontal obtenues à partir des décisions d'approche.

a) Récompense avec solution de sucre $2 \mathrm{~m}$.

b) Récompense avec eau miellée.

Abscisses : nombre des actes d'apprentissage (LA). Sp, choix spontané.

Ordonnées : pourcentage de choix de la croix jaune $\left(\mathbf{W}_{t}\right)$. $n$, nombre des décisions $A$.

Wie die Abb. 9 zeigt, liegt die Sammelmotivation zu Beginn des Lernexperiments bei allen 4 Rassen höher und geht im Verlauf der 10 Lernakte zurück. A.m. carnica und ligustica weisen eine hohe Sammelmotivation auf, die stark zurückgeht; $A$. cerana und $A$.m. lamarckii dagegen haben eine fast gleichmäßige niedrige Sammelmotivation. Dies gilt für beide Versuchsanordnungen. Da die Flugaktivität stark von Witterungsbedingungen abhängt, überlagern diese auch die Sammelmotivation. Dies konnte sich aber nicht bei einer der Rassen stärker auswirken als bei den anderen, da während aller Experimente keine extremen Temperaturunterschiede herrschten (Schwankungen im Bereich 14-25० $\mathrm{C}$ ) und auch keine andauernden Regenperioden eine bestimmte Versuchsserie störten.

Da die Entfernung zum Heimatstock bei den Rassen verschieden war (siehe Methode), könnte man vermuten, daß die indische und ägyptische Biene deshalb eine geringere Suchmotivation zeigten. Tatsächlich flogen die indischen und ägyptischen Bienen während eines 4 Min-Tests meist einmal zum Stock zurück. Sie blieben aber selten länger aus als die anderen beiden Rassen auf weiten Umflügen, die diese regelmäßig während des Tests durchführten.

Man kann daher annehmen, daß Abb. 9 überwiegend die rassenspezifischen Unterschiede in der Sammelmotivation angibt, wenn das auch vorläufig nicht 


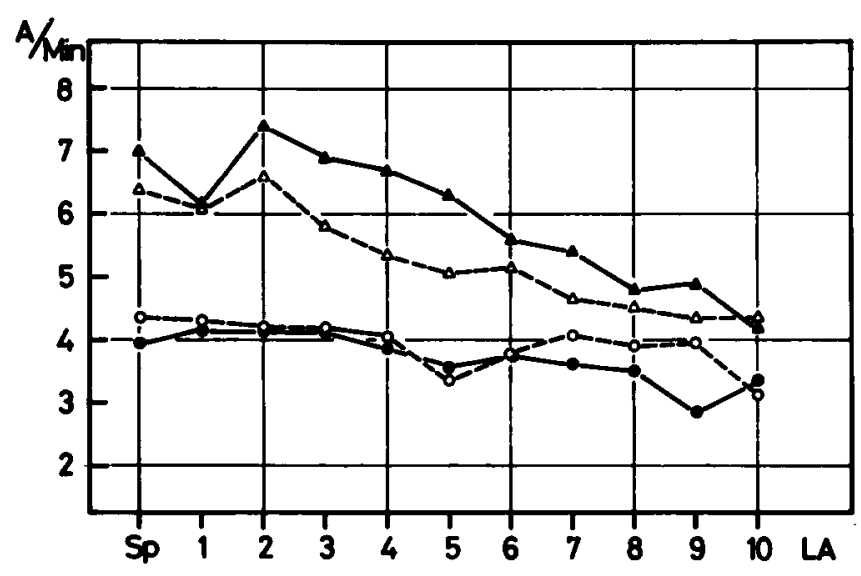

Aвв. 9. - Mittlere Sammelmotivation der 4 Rassen in Abhängigkeit vom Lernverlauf.

Als Sammelmotivation wird die Zahl der Anflüge pro Testminute bezeichnet (Ordinate : A / Min); Abszisse : Zahl der Lernakte (LA), Sp : Spontanwahl. Da sich keine Unterschiede in der Sammelmotivation an der horizontalen und vertikalen Anordnung ergaben, wird hier das Mittel aller A-Entscheidungen an beiden Anordnungen angegeben. - $\mathbf{A}-\mathbf{A}$. $\mathbf{m}$. carnica $(n=6205),-\triangle-A$. m. ligustica $(n=5719),-1-A$. cerana $(n=4450)$, -O- A. m. lamarckii $(\mathrm{n}=\mathbf{4 0 8 3})$.

FIg. 9. - Moyenne de la motivation de récolte des 4 races en rapport avec le déroulement de l'apprentissage.

On désigne par motivation de récolte le nombre des approches par minute de test (Ordonnées : A/Mn). Abscisses : nombre des actes d'apprentissage (LA). Sp, choix spontané. Étant donné qu'il n'y a pas de différence dans les motivations de récolte sur dispositif horizontal ou vertical, on donne ici la moyenne de toutes les décisions A sur les deux dispositifs. $-A-A$. m. carnica $(n=6205),-\triangle-A$. m. ligustica $(n=5719)-0-A$. cerana $(\mathrm{n}=4450)-\mathrm{O}-$ A. m. lamarckii (n. 4083).

mit weiteren Experimenten belegt werden kann. Der Vergleich der Sammelmotivation mit den Lernkurven (Abb. 3, 4, 6) ergibt, daß die beiden Rassen mit besonders raschem Lernverlauf sich durch eine niedrige Wahlmotivation auszeichnen (cerana und lamarckii), während die mit langsamerem Lernfortschritt (carnica und ligustica) wesentlich mehr Entscheidungen im Test machen. Diese quantitativen Angaben belegen den subjektiven Eindruck, daß die mit hoher Aktivität suchenden Rassen auch rasch « ein paar Fehler mehr machen "); es kommt bei ihren schnellen Suchflügen sozusagen nicht so genau darauf an. Dies muß bei der Beurteilung von rassenspezifischen Unterschieden der komplexen Lernstrategie berücksichtigt werden.

\section{2. - Versuchsserie : drei Alternativen im Test}

Bei der Vordressur lernen die Vb in unseren Experimenten ein schwarzes aufgelöstes Kreuz in der Mitte der Dressurplatte. In den bisher geschilderten Experimenten zeigten wir den Versuchstieren diese Marke im Lerntest nicht mehr, sondern nur noch ein gleichgestaltetes blaues und gelbes Kreuz, die gleichweit von der Mitte der Dressurplatte entfernt sind. Auf diese Weise 
können wir prüfen, wie die $\mathrm{Vb}$ das neu eingeführte Merkzeichen (Gelb) lernen. Es bleibt aber unbekannt, welche Rolle der Ort der Dressur (Mitte des Versuchstisches) bei der Wahl der im Test jeweils an einem anderen Ort gebotenen Marken spielt. Da in unserem Versuchsaufbau (großer homogener Hintergrund, weit entfernte Landmarken bis auf den Experimentator, demgegenüber sich die Marken im Test in ihrer relativen Lage von Minute zu Minute ändern) der Ort nur wenig ausgezeichnet ist, markierten wir ihn im Test mit der Lernmarke der Vordressur - dem schwarzen Kreuz. Nach der Vordressur sieht die Versuchsbiene also im 1. Test (Spontanwahl) 3 Alternativen; die gleiche Anordnung findet sie nach jeder Belohnung auf Gelbkreuz (in der Mitte) vor.

Wir gehen bei diesem Versuch davon aus, daß die Tiere neben Farben eine Fülle weiterer Faktoren lernen, vor allem die Form der Marken und ihre räumliche Anordnung. Diese Raumparameter könnten nun ein höheres Gewicht haben, je nach dem, ob die Marken horizontal oder vertikal angeordnet sind, zumal wir wissen, daß bei Bienen die räumliche Meßschärfe von der Anordnung der geometrischen Figuren im Schwerefeld abhängt (WEHNER, 1967). Möglicherweise läßt sich dann auch der Unterschied im Verlauf der Lernkurven bei den beiden Versuchsanordnungen (Abb. 3, 4) erklären. Diese Unterschiede waren bei A.m. carnica und A.m. ligustica am größten; wir führten daher mit diesen beiden Rassen den oben geschilderten Test durch.

Vergleicht man die Lernkurven für Gelbkreuz in der 2- und der 3- Alternativensituation, so finden sich auf der horizontalen Versuchsanordnung nur anfänglich tiefer liegende Lernkurven, nach mehr als 5 Lernakten wird das Schwarzkreuz nur mehr so selten angeflogen, daß sich kaum ein Unterschied mehr festellen läßt. Bei den vertikal angeordneten Marken dagegen liegt die Lernkurve in der 3-Alternativensituation während der ganzen Dressurzeit signifikant tiefer. Um den unterschiedlichen Verlauf während der Dressur deutlicher zu machen, ist in Abb. 10 die Differenz der mittleren A-Wahltendenz im 3- und 2- Alternativentest aufgetragen (Wt-Gelbkreuz im 3-Alternativentest minus Wt-Gelbkreuz im 2-Alternativentest). Je höher die Differenz ist, desto stärker wird das am Belohnungsort liegende Schwarzkreuz gewählt; ist die Differenz Null, wird das Schwarzkreuz nicht mehr gewählt.

Aufgrund der Vordressur wird bei beiden Versuchsanordnungen das Schwarzkreuz anfänglich stark gewählt. Nach Umdressur auf das Gelbkreuz nimmt dann die Wahl des Schwarzkreuzes am Dressurplatz ab. Diese Abnahme setzt sich auf der horizontalen Versuchsanordnung während der 10 LA fort (Abb. 10 a) und verläuft für beide Rassen etwa gleichartig (keine signifikanten Unterschiede). Auf der vertikalen Platte dagegen (Abb. $10 \mathrm{~b}$ ) nimmt für A.m. carnica die Wahl des Schwarzkreuzes vom 3 . LA an nicht mehr weiter ab, bei A.m. ligustica steigt sie sogar wieder erneut an. Der Unterschied zwischen den beiden Rassen bei vertikalen Marken ist nach dem 5. LA gut gesichert. Aus diesem Ergebnis muß man schließen, daß dem Dressurort auf der 
R. MENZEL, H. FREUDEL, U. RÜHL

vertikalen und horizontalen Anordnung ein unterschiedliches Gewicht bei der Orientierung am Futterplatz zukommt und die beiden geprüften Rassen sich in der Gewichtung der wirksamen Raumparameter unterscheiden. Die anfängliche Abnahme der Wahltendenz für Schwarzkreuz ist der Ausdruck des Umlernens auf das Gelbkreuz : Das vorher belohnte Schwarzkreuz wird aufgrund der Belohnungen auf Gelbkreuz immer weniger gewählt. Da aber gleichzeitig das Gelbkreuz stets in der Mitte der Dressurplatte belohnt wird, erfolgt mit zunehmenden Lernakten auf Gelbkreuz eine Verstärkung des Dressurortes. Wegen der geringen Wertigkeit des Dressurortes auf der horizontalen Platte setzt sich hier der Umlernvorgang bei beiden Rassen soweit fort, bis Schwarzkreuz in der Mitte nicht mehr gewählt wird. Die Verstärkung des Dressurortes wirkt sich nicht aus.

Auf der vertikalen Anordnung dagegen hebt sich bei der Kärntnerbiene die abnehmende Wahl des Schwarzkreuzes aufgrund des Umlernvorganges mit der zunehmenden Wahl wegen dem fortschreitenden Lernen des Dressurortes gerade auf (Abb. 10 a). Die Italienerbiene wählt sogar nach mehr als 4 LA den

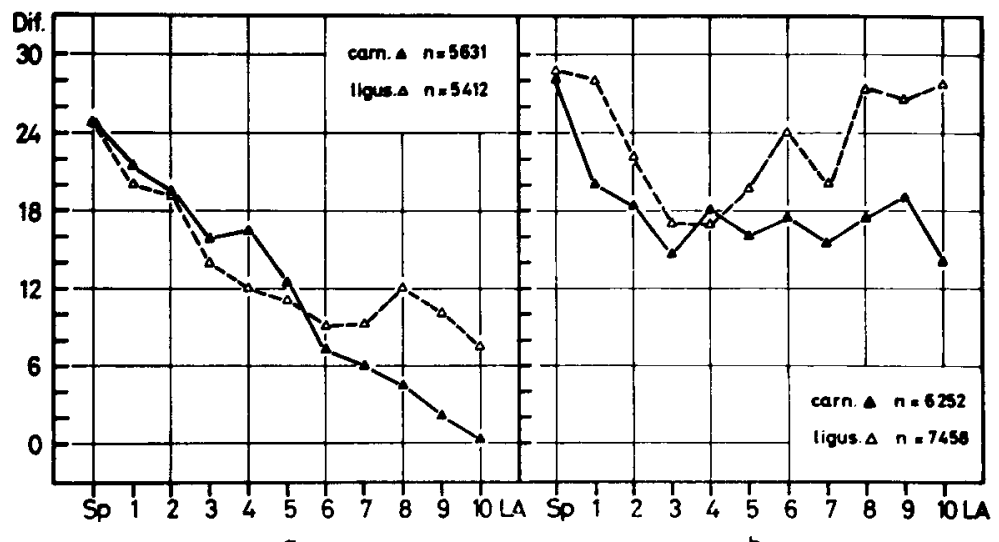

a.

b.

Авв. 10. - Vergleich der mittleren A-Lernkurven von A. m. carnica und A. m. ligustica im 2und 3-Alternativen-Test.

a) an der horizontalen,

b) an der vertikalen Versuchsanordnung. Die Ordinate gibt die Differenz der mittleren Wahltendenz (prozentuale Richtigwahl) im 3- und 2-Alternativen-Test an (Wt im 3-AlternativenTest minus Wt im 2-Alternativen-Test). Abszisse : Zahl der Lernakte (LA), Sp : Spontanwahl. $n$ ist die Zahl der ausgewerteten Entscheidungen.

$\Delta-$ A. m. carnica, $-\triangle-\mathbf{A}$. m. ligustica.

Frg. 10. - Comparaison des courbes d'apprentissage moyennes $A$ de $A, m$. carnica et de $A . m$. ligustica dans les tests à 2 et 3 possibilités de choix.

a) sur dispositif horizontal,

b) sur dispositif vertical.

Ordonnées : différence des tendances de choix moyennes (pourcentage de choix corrects) entre les tests à 2 et 3 possibilités de choix (Wt dans le test à 3 possibilités de choix, moins Wt dans le test à 2 possibilités de choix).

Abscisses : nombre des actes d'apprentissage (LA). Sp, choix spontané. n, nombre des décisions utilisées.

$-\mathbf{A}-=$ A. m. carnica $-\triangle-=$ A. m. ligustica. 
Dressurort wieder zunehmend stärker. Daraus ist zu schließen, daß Raumparameter bei frontalem Anflug für A.m. ligustica ein größeres Gewicht haben als für A.m. carnica. Dies erklärt auch, warum bei $A . m$. ligustica die Lernkurve für vertikale Marken nach dem 5. LA weiter ansteigt (Abb. 4).

\section{DISKUSSION}

Die genetisch fixierte Reaktionsnorm des Lernmechanismus ist ein Faktor von vielen, die auf den Verlauf der Lernkurve einwirken. Da wir Aussagen über diese Reaktionsnorm bei 4 Bienenrassen machen wollen, dürfen wir den Einfluß dieser anderen Faktoren nicht vernachlässigen. Dies ist bei des Analyse der experimentellen Daten versucht worden. Einige äußere Faktoren (z.B. Licht, Temperatur, Belohnungsart, 4 Minuten langer Test) haben sich als unbedeutend herausgestellt; andere zeigten einen großen Einfluß : z.B. die Anordnung der Marken im Schwerefeld, die Markierung des Dressurortes im Test. Alle 4 Rassen lernten vertikale Farbkreuze besser als horizontale. Aber nicht für alle 4 Rassen ist die räumliche Anordnung gleich gewichtig : Für A.m. ligustica spielt sie eine hervorragende Rolle; dies zeigen sowohl die besseren Lernkurven in der vertikalen 2-Alternativensituation (Abb. 4), als auch ein Vergleich der 2- und 3-Alternativensituation (Abb. 10 a, b). A.m. lamarckii dagegen gewichtet die räumliche Anordnung von allen 4 Rassen am geringsten.

In einem Vergleich des Lernverhaltens von A.m. carnica und A.m. ligustica fanden Lindauer (1970), Lauer und Lindauer (1972), dass die Kärntner Biene sich stärker nach der räumlichen Anordnung von Orientierungsmarken in der näheren Umgebung der Futterquelle orientiert als die Italienerbiene. Dies muss mit unseren Befunden nicht im Widerspruch stehen, da wir die Raumparameter der Futterquelle selbst prüften und seit den Untersuchungen von OPFinger (1931) bekannt ist, dass Lernmarken unmittelbar an der Futterstelle und solche in grösserer Entfernung (über $30 \mathrm{~cm}$ ) anders gelernt werden.

Von besonderer Bedeatung erwiesen sich innere Faktoren, wie Sammelmotivation und Suchstrategie. Die beiden Rassen mit den flacheren Lernkurven (A.m. carnica und ligustica) wiesen eine größere und im Verlauf des Lernvorganges abnehmende Sammelmotivation auf, während $A$. cerana und A.m. lamarckii im gesamten Test eine niedrige und weitgehend gleichbleibende Sammelmotivation zeigen (Abb. 9). Als Maß für die Suchstrategie haben wir das Verhältnis der A- und $T+$ S-Entscheidungen ausgewertet. Eine $T+$ S-Lernkurve nimmt bei allen 4 Rassen und in allen Testsituationen einen steileren Verlauf. Eine Änderung der Suchstrategie wird deshalb Folgen für den Verlauf der A-Lernkurve haben müssen. Dies läßt sich in einem Vergleich der beiden rasch lernenden Rassen ( $A$. cerana, $A$.m. lamarckii) mit den weniger schnell lernenden Rassen (A.m. carnica, A.m. ligustica) zeigen. Weiter ergab 
sich, daß die Kopplung zwischen A-Wahltendenz und T + S-Wahltendenz bei den 4 Rassen verschiedenartig vom Lernverlauf abhängt. Dieser Faktor erwies sich als besonders bedeutsam für die Erklärung der A-Lernkurven von A.m. lamarckii an der horizontalen und vertikalen Anordnung.

Die optimale Anpassung des Lernmechanismus als innere Informationsquelle beim Orientierungsverhalten kann nicht darin bestehen, eine belohnte Marke so schnell wie möglich zu lernen und dann nur noch diese anzu fliegen. Die wechselnden Wertigkeiten der Umweltmarken machen die ( Vorsicht » beim Umlernen auf eine neue Marke, aber auch die Fähigkeit, früher belohnte Marken aufzugeben, zu zwei gegenläufigen Forderungen, deren beste Lösung einmal mit der Flug- und Suchaktivität eng zusammenhängt, zum anderen von den Trachtquellen bestimmt wird, auf die eine Rasse sich im Verlauf vieler Jahrtausende eingestellt hat. Die enge Beziehung zwischen rassenspezifischem Lernmechanismus und Suchverhalten konnte in dieser ersten Analyse gezeigt werden.

Über die Unterschiede in den Eigenschaften der traditionellen Trachtquellen der 4 untersuchten Rassen ist uns leider wenig bekannt. A.m. carnica und A.m. ligustica finden in ihrem natürlichen Verbreitungsgebiet (nordöstlich und südlich der Alpen BäнrмanN, 1966) nahezu während der ganzen warmen Jahreszeit eine Großzahl verschiedener Trachtquellen mit häufig wechselnder Nektarproduktion vor. Die ägyptische und indische Biene dagegen bevölkern Gebiete mit sowohl im Laufe des Jahres als auch in ihrer Mannigfaltigkeit beschränkten Nektarquellen. In dieser Situation mag es sich für die Italienerund Kärntnerbiene als günstig herausstellen, sich weniger stark an eine Quelle zu binden, für die beiden anderen Rassen könnte sicherere Wahl und größere Stetigkeit einer Quelle gegenüber von Vorteil sein.

Die nachgewiesenen rassenspezifischen Unterschiede bedürfen jetzt einer genetischen Analyse. Leider stößt das gerade bei der indischen Biene auf Schwierigkeiten, da sich diese beiden Arten nicht miteinander kreuzen lassen (Ruttner und MavL, 1969); eine Kreuzung zwischen den 3 Rassen von A.m. ist aber möglich (RutTnER, 1967). Bisher wurde nur ein Verhaltensmuster der Biene (Nestreinigungsverhalten) mittels Rückkreuzungen untersucht (RoTHENBühler, 1958, 1964). Nye and Mackensen (1965) wiesen nach, daß die Tendenz zum Sammeln von Luzernen-Pollen vererbbar ist. Andere rassenspezifische Verhaltensweisen (z.B. Rhythmik des Schwänzellaufs, Linda uer 1957, Восн 1957, maximale Flugentfernung, v. Frisch 1965, Flugaktivität, Dauer der Paarungs flüge, täglicher Aus flugbeginn und-ende, Hutson 1930, Filmer 1931, Drescher 1969, Aggressivität Dhaliwal 1970, Wertigkeit des Himmelskompasses als Orientierungshilfe, Lauer und Lindauer 1971) wurden beschrieben, bisher aber noch nicht genetisch analysiert.

Unsere Befunde machen wahrscheinlich, daß zentralnervöse Prozesse die Wahlmotivation, die Suchstrategie und die Wahlsicherheit steuern. Wir 
haben aber nicht überprüft, ob sich die Rassen nicht auch in ihren sinnesphysiologischen Eigenschaften - vor allem des optischen Sinnes - unterscheiden. Die unterschiedliche Gewichtung räumlicher Parameter bei A.m. carnica und ligustica, die auch von LAUER und LiNdauer (1971) beschrieben wurde, könnte Differenzen in den ersten Verarbeitungsschritten optischer Information vermuten lassen. Dieser Problemkreis wird weiter untersucht.

Eingegangen im November 1972.

Reçu pour publication en novembre 1972.

\section{RESUME}

Trois races d'Apis mellifica (carnica, ligustica et lamarckii) ainsi que l'espèce $A$. cerana ont été étudiées comparativement du point de vue de leur comportement d'apprentissage.

Dans une première série d'essais (tests à deux possibilités de choix, chapitre 1) des abeilles (Vb) sont soumises individuellement à un pré-dressage sur une croix noire dont les bras sont constitués de figures carrées juxtaposées (fig. 1) et qui est située au milieu d'un plateau de dressage qui peut être horizontal (ler dispositif expérimental) ou vertical ( $2^{\mathrm{e}}$ dispositif expérimental). Après ce pré-dressage on teste leur choix spontané face à deux croix de même forme, l'une jaune et l'autre bleue. Ensuite on accorde la récompense (sirop de sucre) sur la croix jaune. Après chaque récompense sur la croix jaune (acte d'apprentissage, LA) on note pendant 4 minutes le comportement de choix de l'abeille; ce faisant on distingue la décision d'approche (A), de contact (T) et d'atterrissage (S).

Dans une seconde série d'essais (test à trois possibilités de choix, chapitre 2) les deux races Apis mellifica carnica et $A$.m. ligustica sont soumises, comme dans la première série d'essais, à un pré-dressage sur croix noire et elles sont ensuite récompensées sur croix jaune. Durant le test de 4 minutes on propose aux abeilles 3 possibilités de choix : à côté des croix jaune et bleue on laisse la croix noire au milieu du plateau de dressage.

Le progrès dans l'apprentissage est représenté par des courbes d'apprentissage qui sont calculées séparément pour les décisions $A$ et les décisions $T+S$. Les courbes d'apprentissage $\mathbf{T}+\mathbf{S}$ ont, chez les deux races, toujours une pente plus forte que les courbes $\mathbf{A}$. Avec une exception pour les courbes d'apprentissage A d'Apis mellifica lamarckii, les courbes d'apprentissage $A$ pour la croix jaune verticale ont toujours une pente plus forte que pour la croix jaune horizontale. Étant donné que l'accroissement de la tendance de choix A dépend aussi de la fréquence relative des décisions $T+S$ (stratégie exploratoire, fig. 5 ) et du couplage des tendances de choix $A$ et $T+S$ (fig. 6), on a testé pour chaque race l'in fluence de ces facteurs sur le déroulement de l'apprentissage. Le résultat d'une telle analyse combinée de l'apprentissage et du comportement est le suivant :

1) A. cerana apprend le plus rapidement et atteint un haut niveau d'apprentissage. A.m. lamarckii montre un déroulement de l'apprentissage qui a une pente analogue (fig. 3 et 4). Les deux races modifient leur stratégie exploratoire au cours de l'apprentissage de façon telle que des décisions $(T+S)$ plus sûres interviennent plus fréquemment (fig. 5). Le tracé plus plat de la courbe d'apprentissage A d'A.m. lamarckii sur dessins verticaux (fig. 4) s'explique par une modification du couplage entre les tendances de choix $\mathbf{A}$ et $\mathbf{T}+\mathrm{S}$.

2) A.m. carnica et A.m. ligustica donnent des courbes d'apprentissage A plus aplaties (fig. 3,4). Elles n'améliorent pas leur stratégie exploratoire au cours de l'apprentissage (fig. 5). Chez les deux races (particulièrement chez A.m. ligustica) le couplage entre les tendances de choix $A$ et $T+S$, dans les deux dispositifs expérimentaux, se modifient de telle façon que la croix jaune verticale est mieux apprise que l'horizontale (fig. 6).

3) La motivation du choix (approches par minute de test) est différente chez les 4 races (fig. 9) : elle est élevée chez $A$.m. ligustica et carnica au début de l'expérience d'apprentissage puis elle recule fortement. Chez $A$.m. fasciata et $A$. cerana la motivation du choix est plus faible et baisse peu.

4) Une comparaison du comportement d'apprentissage d'A.m. carnica et ligustica dans 
R. MENZEL, H. FREUDEL, U. RÜHL

les séries d'essais 1 et 2 montre que les paramètres spaciaux ont pour les deux races une plus grande importance dans le dispositif vertical que dans le dispositif horizontal. A.m. ligustica apprend encore mieux la position dans l'espace des signaux d'apprentissage qu'A.m. carnica.

5) La valeur informative des courbes d'apprentissage dans les analyses comparatives d'apprentissage est discutée. Il apparaît que les courbes d'apprentissage ne peuvent apporter d'information sur le mécanisme d'apprentissage que dans la mesure où la tendance de choix est connue avant le début de l'apprentissage (choix spontané) et que, si l'on tient compte du fait que le comportement de choix au cours de l'apprentissage et dans différentes situations. expérimentales est modifié qualitativement; cette modification doit être prise en considération dans l'interprétation des résultats.

6) Le point de vue écologique est rapidement commenté.

\section{LITERATUR}

B̈̈hrmann R. Merkmalstudien und intraspezifische Verwandtschaftsverhältnisse bei Apis mellifica. Z. Bienenforschung 8, 17-32 (1965).

Bocr R. Rassenmäßige Unterschiede bei den Tänzen der Honigbiene A. mellifica. Z. vergl. Physiol. 40, 289-320 (1957).

Drescher W. Die Flugaktivität von Drohnen der Rassen Apis mellifica carnica und Apis mellifica ligustica in Abhängigkeit von Lebensalter und Witterung. Z. Bienenforsch.9, $151-170$ (1969).

Dhaliwal S. Interspecific relation between A. cerana and A. mel. mellifera. J. Apicultr. Res. 9, (2), 19-21 (1970).

Filmer R. Bee investigation. N. J. State Exp. Sta. 52 nd Ann. Rep., 228-238 (1931).

Frisch K. von. Tanzsprache und Orientierung der Bienen. Springer, Heidelberg, Berlin (1965).

Hertz M. Die Organisation des optischen Feldes bei der Biene. III. Z. vergl. Physiol. 14, 629-674 (1931).

Hutson R. Bee investigantions. N. J. State Agr. Exp. Sta. 51 Ann. Rep. 176-182 (1930).

Lindauer M. Sonnenorientierung der Biene unter der Äquatorsonne und zur Nachtzeit. Naturwissenschaften 44, 1-6 (1957).

LiNdaver M. Lernen und Gedächtnis, Versuche an der Honigbiene. Naturwissenschaften 57, 463-467 (1970).

Lauer J. und Lindauer M. Genetisch fixierte Lerndispositionen bei der Honigbiene. Inf. Org. $1,1-88(1971)$.

Menzel R. Untersuchungen zum Erlernen von Spektralfarben durch die Honigbiene. Z. vergl. Physiol. 56, 22-62 (1967).

Menzed R. Das Gedächtnis der Biene für Spektralfarben I. Kurzzeitiges und Langzeitiges Erinnern. Z. vergl. Physiol. 60, 82-102 (1968).

Menzet R. Das Gedächtnis der Honigbiene für Spektralfarben. II. Umlernen und Mehrfachlernen. Z. vergl. Physiol. 63, 290-309 (1969).

Menzel R. und Erber, J. The In fiuence of the quantity of reward on the learning performance in honeybees. Behav. (Leid.) 41, 27-42 (1972).

Nye W. P. and O. Mackensen. Selective breeding of honeybees for alfalfa pollen : fifth generation and backcrosses. J. Apicultr. Res. 7, 21-27 (1968).

Opfinger Eurs., 1931. Über die Orientierung der Bienen an der Futterquelle. Z. vergl. Physiol. $15,237-272$.

Rothenbübler W. Genetics of behaviour differences in honeybees. Proc. 10th Int. Congr. Genet. 2, 252-261 (1958).

Rothenbühler W. Auch das Verhalten ist erblich festgelegt. Archiv Bienenkunde 41, 22-32. (1964).

Ruttner F. Die Zuchtauslese bei der Biene. Ehrenwirth Verlag, München, 2. Auf. (1967).

Ruttner F. und Maul V. (1969). Die Ursache der Kreuzungsbarriere zwischen Apis mellifica L. und Apis cerana Fabr. Verh. 22. Intern. Kongr. Bienenzucht, 515-516, 561.

WaErden B. L. van der und E. Nievergelt. Tafeln zum Vergleich zweier Stichproben mittels x-Test und Zeichentest. Springer, Heidelberg, Berlin (1956).

Wehner R. Zur Physiologie des Formensehens bei der Honigbiene. II. Winkelunterscheidung an Streifenmustern bei variabler Lage der Musterebene im Schwerefeld. Z. vergl. Physiol. $55,145-166$ (1967). 